Results Tp's BamA (TP0326) is the central component of a hybrid BAM system in which POTRA1-5 interacts with the DUF domain of TamB (TP0325). Although Tp lacks lipopolysaccharide, its genome encodes a nearly complete LPS transporter apparatus. The LptD ortholog (TP0515) contains a large unstructured C-terminal domain, which models LptE-like inside the $\beta$-barrel. Tp encodes four 8 -stranded $\beta$-barrels, each containing positively charged extracellular loops that could contribute to pathogenesis. Surprisingly, Tp encodes five FadL orthologs, all of which display features (i.e., hatch domain and NPA motifs) that are characteristic of Gram-negative FadLs. Three (TP0548, TP0859 and TP0865) have $\alpha$-helical extensions that could interact with periplasmic components. The Tp genome encodes Mac and RND efflux pumps that presumably achieve combinatorial diversity via co-expression of four paralogous OMFs. Lastly, we confirmed the bipartite membrane topology of Tprs using SAXS to solve solution structures for MOSPN domains of TprK and MOSP.

Conclusions The Tp Nichols OMPeome provides a structural framework to (i) elucidate Tp's enigmatic parasitic strategies, (ii) identify targets of natural immunity, and (iii) interrogate candidate vaccinogens.

\subsection{ANTIGENIC VARIATION IN A PRIMATE MODEL OF PERSISTENT MYCOPLASMA GENITALIUM INFECTION}

G Wood* , L Aguila, D Patton. University of Washington, Seattle, USA

\subsection{6/sextrans-2021-sti.52}

Background Mycoplasma genitalium persists in the genital tract for months despite the presence of specific antibody that reacts with the immunodominant $\mathrm{MgpB}$ and $\mathrm{MgpC}$ adherence proteins. MgpB and $\mathrm{MgpC}$ undergo antigenic variation via recombination between the mgpBC expression site and archived variable sequences present in nine MgPar sites located around the genome. The objective of this study was to determine if antigenic variation occurs in our pig-tailed macaque model of persistent cervical infection, if variants arise coincident with the appearance of antibodies specific for $\mathrm{MgpB}$ and $\mathrm{MgpC}$, and if variation correlates with persistence. Methods MG was cultured from persistently infected primates over 18 weeks. Whole genome sequencing was performed on these MG cultures using Nanopore sequencing technology then compared to the MG reference genome and to in vitro passaged bacteria.

Results Antigenic variants arose in the variable regions of $\mathrm{MgpB}$ and $\mathrm{MgpC}$ in infected primates but not during in vitro passage in broth. Variants were abundant in primates with persistent infection, and dynamic with different variants predominating at different time points. The sequence changes observed were consistent with reciprocal exchange of variable regions between the expression site and MgPars. Antigenic variants were not detected in a primate that cleared the infection after four weeks, suggesting that variation is required for persistence.

Conclusion Antigenic variation of the $\mathrm{MG} \mathrm{MgpB}$ and $\mathrm{MgpC}$ proteins occurs frequently in vivo and is associated with persistence in the genital tract of female pig-tailed macaques. Future work will measure antibody reactivity to specific variable and conserved regions within the MgpB and MgpC proteins to correlate the appearance of antibody to specific sequences with clearance from the genital tract.

\section{6 A CHLAMYDIA TRACHOMATIS INCLUSION MEMBRANE PROTEIN BLOCKS INTERFERON GAMMA STIMULATED IMMUNITY IN HUMANS AND NONHUMAN PRIMATES}

M Fernandez*. University of Washington, Seattle, USA

10.1136/sextrans-2021-sti.53

Background Epithelial cells that are stimulated with interferon gamma (IFN $\gamma$ ) carry out a potent immune response termed cell-autonomous immunity. While Chlamydia trachomatis is susceptible to murine cell-autonomous immunity, it evades this response in humans. The virulence factors underlying this evasion are unknown. A better animal model is needed to evaluate the importance of this defense pathway against C. trachomatis infection.

Methods We leveraged a novel library of C. trachomatis x C. muridarum chimeras to identify candidate virulence factors. We assayed chimeras and follow-up mutants for their ability to evade inclusion ubiquitination in IFN $\gamma$-primed human cells. Growth restriction assays were performed in human cells in the presence or absence of IFN $\gamma$. We isolated primary fibroblasts from a macaque skin biopsy to test whether a similar response is induced by IFN $\gamma$-stimulation in the nonhuman primate (NHP) model.

Results The inclusion membrane protein CT135 is required for evasion of cell-autonomous immunity. Organisms lacking a functional copy of this gene failed to block ubiquitin recruitment to their inclusions in IFN $\gamma$-primed human and NHP cells. This ubiquitination was associated with a severe growth defect in vitro. Retrospective analysis of a NHP study revealed an inoculum containing a CT135 mutant had poor fitness in vivo. We clonally isolated the mutant and confirmed it was susceptible to cell-autonomous immunity.

Conclusion A historic paucity of genetic tools has hindered the identification of virulence factors that facilitate Chlamydia trachomatis pathogenesis. By leveraging a novel library of mutants, we identified a virulence factor that facilitates chlamydial subversion of cell-autonomous immunity in human and NHP cells. While this C. trachomatis gene is nonfunctional in the mouse model, our reevaluation of a previous study suggests it is required for in vivo survival in NHP. This work underscores the importance of NHP as model organisms to study chlamydia immunity.

\subsection{AT LONG LAST: GENETIC ENGINEERING OF TREPONEMA PALLIDUM SUBSP. PALLIDUM, THE SYPHILIS SPIROCHETE}

${ }^{1,2} \mathrm{~L}$ Giacani*, ${ }^{1} \mathrm{E}$ Romeis, ${ }^{1} \mathrm{~L}$ Tantalo, ${ }^{3} \mathrm{~N}$ Liebermann, ${ }^{3} \mathrm{Q}$ Phung, ${ }^{3} \mathrm{~A}$ Greninger. ${ }^{1}$ Department of Medicine, Division of Allergy and Infectious Diseases, University of Washington, Seattle, USA; ${ }^{2}$ Department of Global Health, University of Washington, Seattle, USA; ${ }^{3}$ Department of Laboratory Medicine, University of Washington, Seattle, USA

\subsection{6/sextrans-2021-sti.54}

Background The recently described method for in vitro cultivation of the syphilis agent, Treponema pallidum subsp. pallidum (T. pallidum), paved the way to transformation experiments to genetically engineer this pathogen. The demonstration that genetically modified syphilis treponemes are attainable would represent a milestone in syphilis research, destined to revolutionize our approach to understand the mechanisms behind T. pallidum success as a pathogen. Here, 